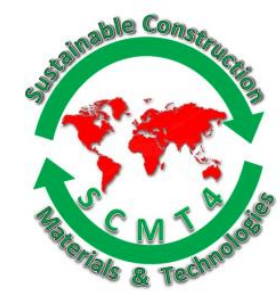

SCMT4

Las Vegas, USA, August 7-11, 2016

\title{
Mesoscopic Model of Volume Changes Due to Moisture Changes in Hardend Cement Paste
}

\author{
Yuka Morinaga ${ }^{1 a}$, and Toyoharu Nawa ${ }^{1 b}$ \\ ${ }^{I}$ Division of sustainable resorces; Hokkaido university, JAPAN; Department of urban environment., \\ ${ }^{1 a}$ Email: <glico611@eis.hokudai.ac.jp>, ${ }^{1 b}$ Email:<nawa@eng.hokudai.ac.jp>.
}

\begin{abstract}
Variation in moisture content in cement paste matrix of concrete occur its volume changes. This drying shrinkage arises sometimes cracking, which induce one of serious problems about concrete durability. Schiller et al proposed the mesoscopic model of volume changes due to moisture variation in porous materials having cylinder shape pores with thermodynamic approach. Their theoretical estimations on the volume changes have a well agreement with experimental data for vycol glass, however they do not describe the behavior of hardened cement paste, in particular hysteresis of desorption and adsorption processes. We focus on the fact that microstructure in cement paste matrix consists of slit like pores as well as cylindrical shape pores. Thus in this paper, we propose a new mesoscopic model of volume change in porous materials having slit shape pores with thermodynamic approach. We discuss first the model of slit shape pores, of which distance between two flat plates is fixed. The model disagrees with experimental hysteresis loop of volume change for hardened cement paste. Second, we proposed the volume-change model of slit shape pores consisted with mobile plates. The slit shape pores model with mobile plates can reproduce the experimental hysteresis loop of volume change for hardened cement paste.
\end{abstract}

\section{INTRODUCTION}

A key issue in materials science is a quantitative evaluation of microstructure to make a mechanistic link between processing and properties. In cement chemistry, the microstructural feature of the larger pore such as capillary pore has been well understood. Thus one can control their porosity by varying a water to cement ratio, and produce concrete with an expected strength. Hence, model that links the properties to specific microstructural features should be necessary for the quality control, degradation assessment, and design of new cement-based materials. The microstructure of cement paste contains water in the pores. The water is evaporated as decreasing an ambitious relative humidity, resulting in the drying shrinkage of concrete. If drying shrinkage is beyond a given extent, cracking causes in concrete, which results in the reduction of durability.

Schiller et al. [Shiller et al., 2008] has proposed a meso-scale model that can describe the expansion and contraction of the elastic porous body having a cylindrical pore. In this model it was reproduced with much higher accuracy experimental data of vycor glass. However, their model for cylindrical pores could not provide an adequate explanation for the hysteresis in adsorption-desorption isotherm of hardened 
cementitious materials. Cement paste has multi-scale pore structure, and show hysteresis over the whole range of relative humidity (RH) from $0 \%$ to $100 \%$. Hysteresis is also different between vycor glass and cement paste. The swelling of hardened cement paste (hcp) during the adsorption process is smaller than shrinkage of hcp at the first desorption process, while vycor glass exhibits the reverse tendency. The same tendency of hysteresis is also observed for clays. For cement paste, the key role of water in C-S-H molecular layers and other possible molecular-scale spaces is further evidenced by the drying shrinkage behavior. A self-consistent model of both sorption and drying shrinkage that captures the hysteresis and explains the difference between cement paste and Vycor glass is still not established.

Burgess et al. [1989] pointed out that the slit pore model can be explained more than cylindrical pore model if a pronounced hysteresis is observed. Structural models of C-S-H suggest the existence of slit pores in hardened cement paste. In this study, we focus on to build thermodynamic model of drying shrinkage of slit type pores to preciously predict the drying shrinkage behavior, in particular the hysteresis of adsorptiondesorption process.

\section{Thermodynamic model of slit pore between two immobile parallel plates}

First let consider the slit-type pore, which consists of two parallel plates and the distance between two plates is fixed. The size of plate is $a(\mathrm{~nm})$ in length, $a(\mathrm{~nm})$ in width and $t(\mathrm{~nm})$ in thickness, as shown in figure 1. If the slit-type pores is filled partially by a condensed liquid that forms a hydrophilic thick water film on a solid surface plate. When the separation distance between the surface of absorbed water layer is $d(\mathrm{~nm})$, there is the water film with the thickness of $t=h_{0}-d$ on the wall in slit-type pores. Within the frame of Gibbs surface diving theory, the grand canonical potential in such a system can be expressed as

$$
\frac{\Omega(t, h)}{a^{2}}=2 \gamma_{s l}+2 \gamma_{l v}-\frac{2 t}{V_{m}} R T \ln \left(\frac{p}{p_{0}}\right)+\frac{2 \omega(t, \lambda)}{a^{2}}
$$

where $\Omega_{\text {slit }}(\mathrm{J})$ is the free energy applied to the slit-shaped pores, $\gamma_{s l}\left(\mathrm{~J} / \mathrm{m}^{2}\right)$ and $\gamma_{l v}\left(\mathrm{~J} / \mathrm{m}^{2}\right)$ are interfacial tension of solid-liquid interface and liquid-vapor interface, $V_{\mathrm{m}}\left(\mathrm{m}^{3} / \mathrm{mol}\right)$ is the molar volume of water, $R$ is the gas constant, $T(\mathrm{~K})$ is absolute temperature and $P / P_{0}$ is the relative vapor pressure $(-), \lambda$ is decay length, $\Pi_{0}$ $\left(\mathrm{N} / \mathrm{m}^{2}\right)$ is the disjoining pressure. The first and second terms on the right-hand side in equation (1) show free energy due to interfacial tension in solid-liquid interface and liquid-vapor interface respectively. The third term shows the chemical potential of liquid-like water in the pore. The fourth term expresses the free energy due to the disjoining pressure. The disjoining pressure is the excessive pressure manifested in the liquid in the contact spacing upon its thicking or thicking. The idea of disjoining pressure in thin boundary films was first introduced by Deryagin and Kusakov [ ,1936], determined for a flat surface. To a first approximation, the disjoining pressure consists of the following components [Deryagin and Churaev, 1984]

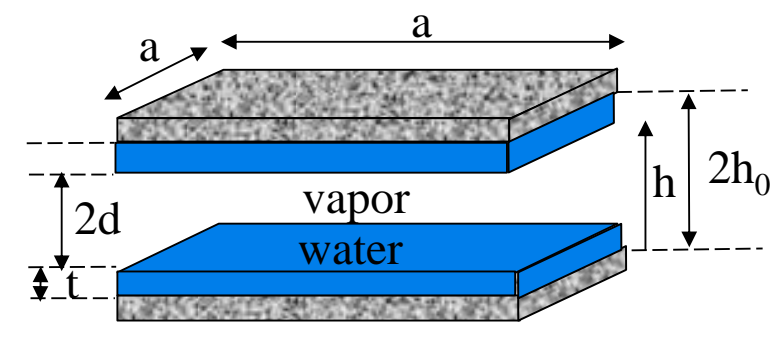

Figure 1 .Schematic of slit-like pore with immobile plates

$\Pi(h)=\Pi_{m}(h)+\Pi_{c}(h)+\Pi s(h)$ 
where $\Pi_{\mathrm{m}}(h)$ is the molecular component produced by the dispersion interaction of the solid surface through a thin liquid film, $\Pi_{\mathrm{c}}(h)$ is the electrostatic component resulting from overlapping of diffusive ionic layers of charged particle surfaces, and $\Pi_{\mathrm{s}}(h)$ is the structural component of disjoining pressure caused by the overlapping of adsorption liquid layers with the modified structure. Thus the free energy due to disjoining pressure $\omega(h)$ are controlled by the input of above-mentioned surface attractive and repulsing forces of various origins. Several researchers have been proposed the different type formulas to represent the free energy due to disjoining pressure, Marceja and Radic[,1976] gave the following two-parametric expression:

$$
\frac{\omega(t, \lambda)}{a^{2}}=\lambda \Pi_{0} \frac{\exp (-t / \lambda)+1}{2 \sinh (t / \lambda)}
$$

where $\lambda$ is the decay length and $\Pi_{0}\left(\mathrm{~N} / \mathrm{m}^{2}\right)$ is disjoining pressure.

Approximation for the free energy of disjoining pressure $\omega(h)$ can be obtained from the experimental data about the thickness of water film on cementitious material surface. Badman et al. [, 1981] quantified the statistical thickness of adsorbed water film on two nonporous $\mathrm{C}_{2} \mathrm{~S}$ and $\mathrm{C}_{3} \mathrm{~S}$ at $293^{\circ} \mathrm{K}$. The thickness of water film $t$ is determined from the equation $\partial \Omega / \partial t=0$, and is represented as a function of the reduced vapor pressure $p / p_{0}$. On the basis of our fitting we obtain the values of the parameters to be used for modeling water adsorption on hardened cement paste surface: $\Pi_{0}=20 \times 10^{6} \mathrm{~N} / \mathrm{m}^{2}, \lambda=1 \mathrm{~nm}$. Figure 2 shows the thicknesses of adsorbed water films for interlayer distance $h_{0}=1,2.5$ and $5 \mathrm{~nm}$ at $20^{\circ} \mathrm{C}$ as a function of different ambient relative humidity. Moreover it can be also seen that in $\mathrm{RH}$ of $90 \%$ or less the thickness of absorbed water film does not differ by the interlayer distance. However, at RH of $98 \%$, the thickness of adsobed water film for $h_{0}=2 \mathrm{~nm}$ is lower than those for $h_{0}=2.5$ and $5 \mathrm{~nm}$ since the slit-like pore with $\mathrm{h}_{0}=$ $1 \mathrm{~nm}$ is completely filled with water.

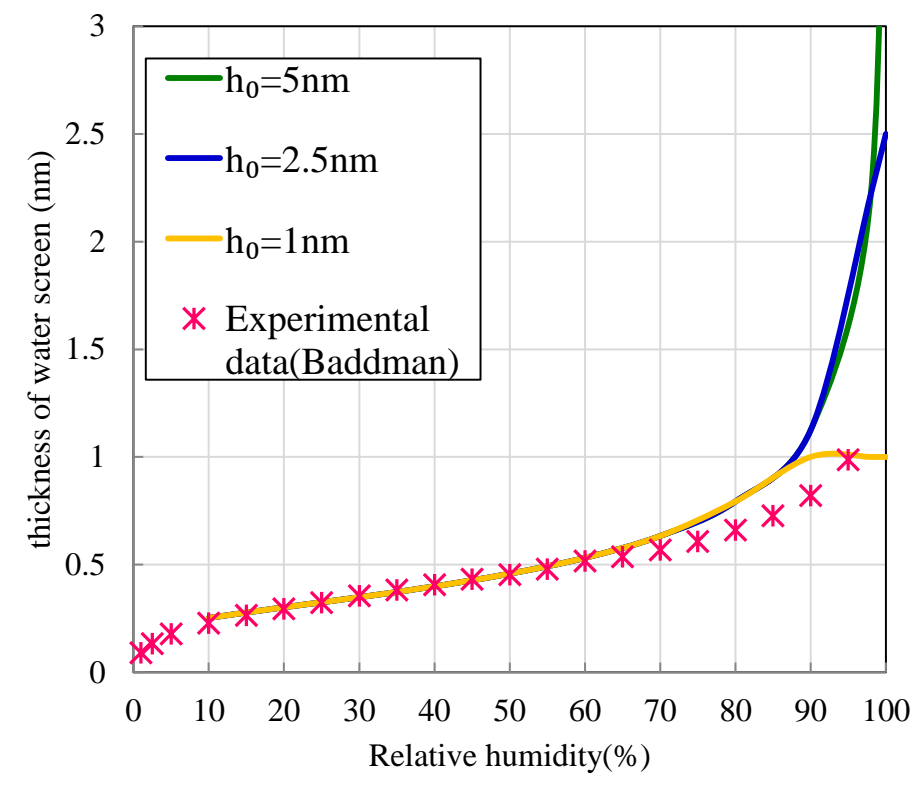

\section{Figure 2. Theoriecal adsorbed water film compared to experimental data published by Baddman[,1976]}

Water film absorbed on the plate wall is stabile when the grand canonical potential is satisfied with the 
following conditions.

$\frac{\partial^{2} \Omega}{\partial t^{2}}>0$ and $\frac{\partial \Omega}{\partial t}=0$

The equation $\partial^{2} \Omega / \partial t^{2}=0$ localizes the threshold of capillary condensation, which is a characteristic feature of the adsorption branch. The pathways of adsorption and desorption in a pore are different. For desorption branch, the condensate of a completely filled pore remains stable as long as the condition of $\Omega$ $(t, h)>\Omega(h, h)$ is satisfied. If $\Omega(h, h)=\Omega(t, h)$ for adsorption branch, completely filled pores are in equilibrium with partly filled ones, and if $\Omega(h, h)>\Omega(t, h)$ the adsorbed water films remain stable, so-call capillary evaporation occurs. For desorption branch, the condensate of a completely filled pore remains stable as long as the condition $\Omega(t, h)>\Omega(h, h)$ is satisfied. If $\Omega(h, h)=\Omega(t, h)$ for adsorption branch, completely filled pores are in equilibrium with partly filled ones, and if $\Omega(h, h)>\Omega(t, h)$ the adsorbed water films remain stable, so-call capillary evaporation occurs.

We can employ the equations (1) and (2) to calculate water vapor adsorption/ desorption isotherm on a slit pore with two immobile parallel plates $\left(\gamma_{s l}=0.2 \mathrm{~J} / \mathrm{m}^{2}, \quad \gamma_{l v}=0.072 \mathrm{~J} / \mathrm{m}^{2}, \Pi_{0}=20 \times 10^{6} \mathrm{~N} / \mathrm{m}^{2}, \quad \lambda=1 \mathrm{~nm}, a=4 \mathrm{~nm}\right.$, $\left.h_{0}=2.5 \mathrm{~nm}\right)$. The fraction the total pore volume which is filled by condensate can be expressed as

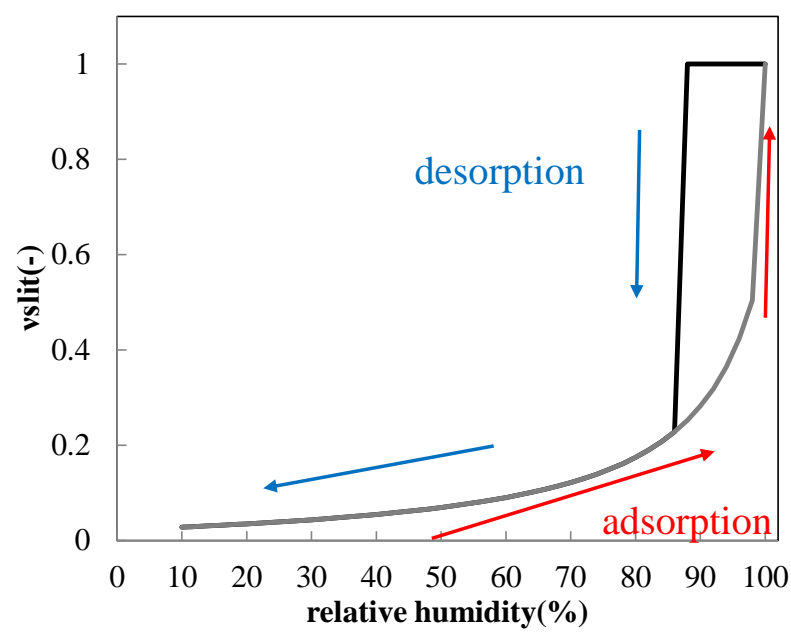

Figure 3. Theoretical water vapor isotherm calculated by slit-like pore model between two immobile parallel plates with $h_{0}=5 \mathrm{~nm}$

$v=\frac{t}{h}$

Figure 3 gives the calculated isotherms at $298 \mathrm{~K}$. The distance between two plates corresponds to the relative pressure $p / p_{0}=0.88$. The adsorption branch in isotherm correspond to relative pressure below 0.99 is the same as the adsorption branch. This means that in this region, the adsorbed water film becomes stable compared to water condensed in slit pore. In contract, the capillary condensation occurs at very high relative humidity. For slit pore with $h_{0}=1 \mathrm{~nm}$, the capillary condensation occurs at RH of $98 \%$, for slit pore with $\mathrm{h}$ $=5 \mathrm{~nm}$ it occurs at RH of $99 \%$. Therefore it can be concluded that a hysteresis results in a region around the threshold value of capillary condensation.

The pressure $P$ that a condensate exerts on a pore wall is quantified as the derivative of the grand canonical 
potential $\Omega$ of the adsorbed phase with respect to the pore volume $V$ at fixed temperature $T$ and adsorbate chemical potential $\mu$ :

$$
P(t, h)=-\frac{1}{a^{2}}\left(\frac{\partial \Omega(t, h)}{\partial t}\right)
$$

Hence volume changes with a slit-like pore with two immobile plates can be written as

$$
\frac{\Delta V}{V}=\frac{P(t, h)}{K_{\text {eff }}}
$$

where $K_{\text {eff }}$ is the effective elastic modulus of the cement paste, which responds to interior tensions produced by pores.

Figure 4 shows the volume changes of slit pore correspond to figure 3 the thickness $t$ of water film below $\mathrm{RH}$ of $88 \%$ for desorption branch and $\mathrm{RH}$ of $99 \%$ for adsorption branch is not

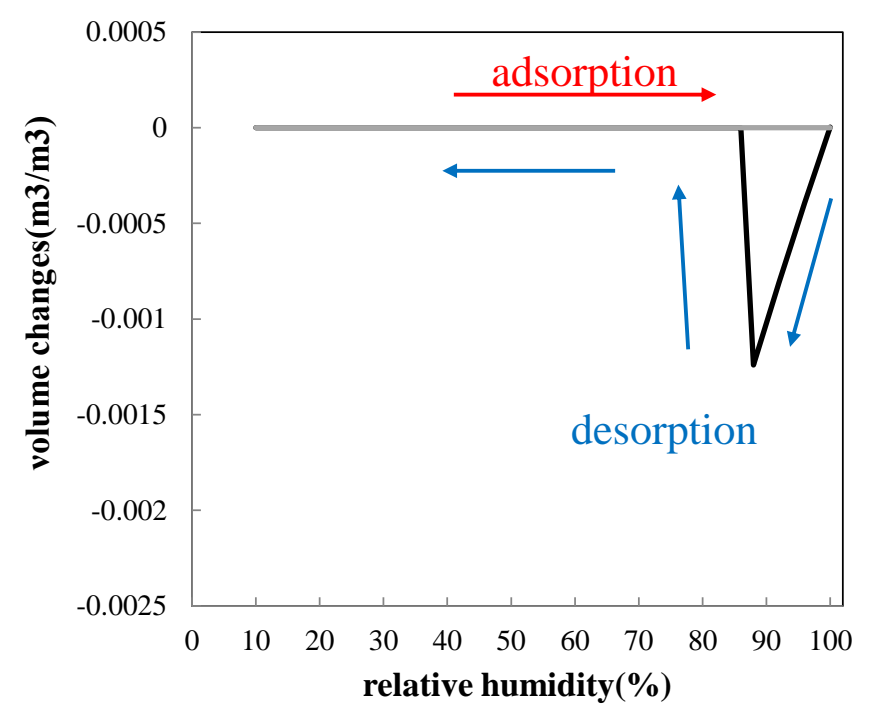

Figure 4. Theoretical volume change and calculated by slit-like pore model with immobile two parallel plates

dependent on distance $h$ between two plates, thus the grand canonical potential becomes constant.

From equation (6), it is obvious that the pressure $P$ becomes zero in these regions in adsorption and desorption processes, resulting in no volume changes.

For desorption branch, beyond $\mathrm{RH}$ of $88 \%$ the grand canonical energy decreases with the relative pressure, and at RH of $88 \%$ the shrinkage dramatically reduces to zero. Hence the volume changes of the slit pore between two immobile parallel plates show the hysteresis loop, but their relation between adsorption and desorption processes is reversed against those for hardened cement paste as well as a cylindrical pore. Furthermore the predicted maximum drying shrinkage even at maximum value is $1.24 \times 10^{-3}$ at $\mathrm{RH}$ of $88 \%$, which is very small compared to typical values of hardened cement paste. These results imply that the proposed slit-pore model consisted of two immobile plates cannot be described the hysteresis of the adsorption-desorption isotherms and volume change of cement paste. 


\section{Thermodynamic model of slit-pore between two mobile parallel plates}

Nonat [,2006] indicated from the AFM observation that CSH gel is assembles of the nanoparticles. These $\mathrm{CSH}$ particles are combined not by the chemical bond, but physical bond. This lead to the hypothesis that two parallel plates that configure slit pores can move freely each other. Here we shall consider is the more realistic slit-like pore, which consist of two parallel plates and the distance between two plates is variable (see Figure 5).

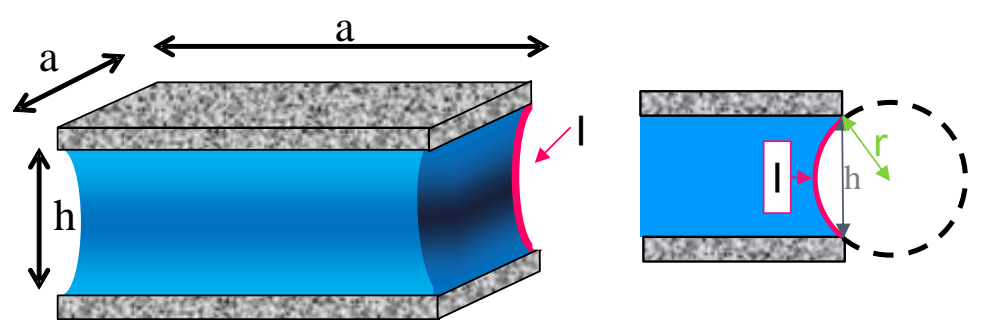

Figure 5. Schematic of slit-like pore with mobile parallel plates

In this system the liquid-vapor interface is present in the four sides of the slit pore since the pore is always completly filled with water. It should be noted that the free energy due to disjoining presure become a function of two parameters of $h$ and $\lambda$. Approximating the surface area of liquid-vapor interface with $2 a h$, the grand chanonical potential or a slit-like pore with two mobile plates can be rewritten as:

$$
\frac{\Omega(h)}{a^{2}}=2 \gamma_{s l}+4 l(p)\left(\frac{l}{a}\right) \gamma_{l v}-\frac{h}{V_{m}} R T \ln \left(\frac{p}{p_{0}}\right)+\frac{\omega(h, \lambda)}{a^{2}}
$$

$l(p)$ is the surface area of liquid/vapor interface, which is represented as a function of curvature radius of liquid/vapor interface (meniscus) $r$ and the width of slit pore $h$, as shown in figure 5. In addition, the Kelvin equation can describe the impact of relative vapor pressure on a curvature radius $r$,

$\ln \left(\frac{p}{p_{0}}\right)=-\frac{2 \gamma_{l} V_{m}}{r R T}$

where $r$ is the curvature radius of liquid/vapor interface in slit pore.

The critical thickness that a water film in a slit pore losses stability, can be calculated from the following conditions:

$$
\partial \Omega / \partial h=0 \text { and } \partial^{2} \Omega / \partial h^{2}>0
$$

We employed the equations (7) and (9) to calculate water vapor adsorption/desorption isotherm on a slit pore with two immobile plates. As for the parameters for disjoining pressure we use the values obtained by fitting with the Baddman's data[Baddman, 1981] about the relation between the statistical thickness of adsorbed water film and relative vapor pressure.

Based on the thermodynamics, the expantion of slit pore due to an increase of the relative humidity from $p / p_{0}$ to $(p+\Delta p) / p_{0}$ requires the work that put the water of increased volume $\Delta V$ into the slit pore. hpc increases it in volume increment in slit pores. This work should be done against the interaction force, socalled disjioing pressure acting between the two parallel flat plates, which is given by the following 
equation:

$w(h)=\int_{h}^{h+\Delta h} \frac{\partial \omega(h)}{\partial h} d h$

where $\Delta h$ is the increment of thickness of slit pore due to change in the relative humidity from $p / p_{0}$ to $(p+\Delta p)$ $/ p_{0}$.

It should be noted that, in the grand canonical potential of the state at relative humidity of $(p+\Delta p) / p_{0}$ is equal to the sum of grand canonical potential of the state at relative humidity of $p / p_{0}$ and the excess work expressed in equation (9). Therefore, we can calculate the the pressure increment $\Delta p$ corresponding to the increase of the thickness of slit pore using the following equation.

$-\frac{h+\Delta h}{V_{m}} R T \ln \left(\frac{P}{P_{0}}\right)+\int_{h}^{h+\Delta h} \frac{\partial \omega(h)}{\partial h} d h+4 \gamma_{l v}\left(\frac{l(P)}{a}\right)=-\frac{h+\Delta h}{V_{m}} R T \ln \left(\frac{p+\Delta p}{p_{0}}\right)+4 \gamma_{l v}\left(\frac{l(P+\Delta P)}{a}\right)$

For the shrinkage in the desorption process, we should consider the work to extract the water of $\Delta V$ from the slit pore, and the canonical potential of the state at relative humidity of $(p-\Delta p) / p_{0}$ is equal to that to substract the excess work represented in equation (9) from the grand canonical potential of the state at relative humidity of $p / p_{0}$. Further, the volume changes are calculated using equations (6) and (7). The water vapor isothem and volume changes for the slit pore model with two immobile plates is shown Figures 6 as a function of different relative humidity. The theoretical adsorption/desorption isotherm and swelling/shrinkage isotherm for slit pore illustrated in Figs. 8 provide a fairly good reproduction of the experimental data as shown in Figure 8 published by Feldman and Sereda [,1968] for cement paste. The sorption isothrm show hysteresis over the whole range of $\mathrm{RH}$ from $0 \%$ to $100 \%$. The volume at a given $\mathrm{RH}$ is smaller wetting than during, when the experiment is performed over the entire range of RH. However, as for the magnitude, adsorption/desorption isotherm and swelling/shrinkage isotherm for slit pore seems to behave differently. This contributes to additional causes such as a specific ink bottle geometry of pores and network effects, which enhances hysteresis effects.
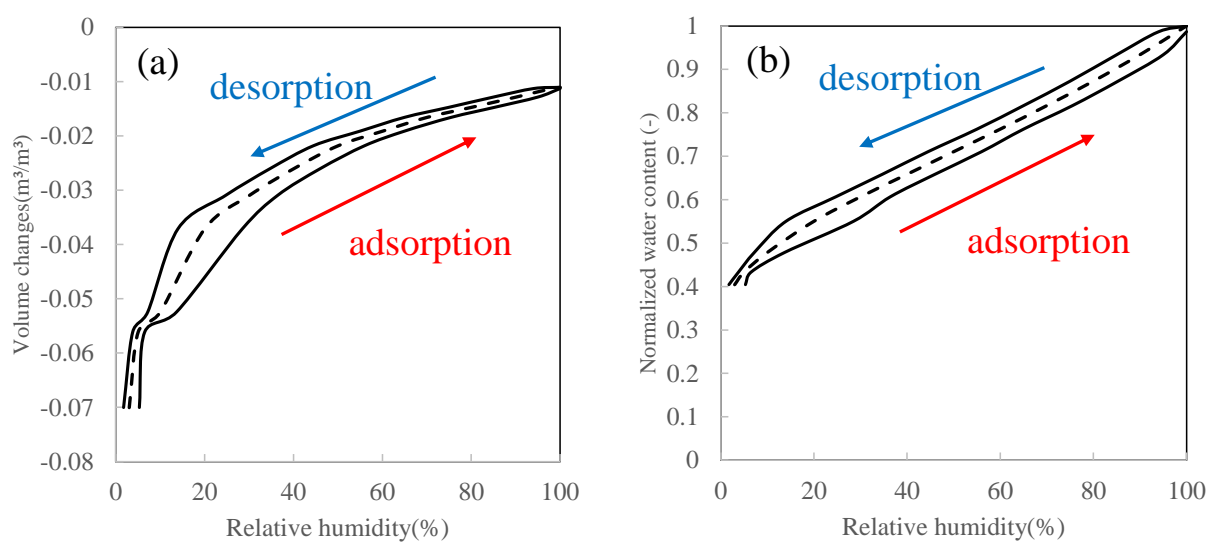

Figure 6. Theoretical (a)volume change and (b)water vapor isotherm calculated by slitlike pore model with mobile two parallel plates 

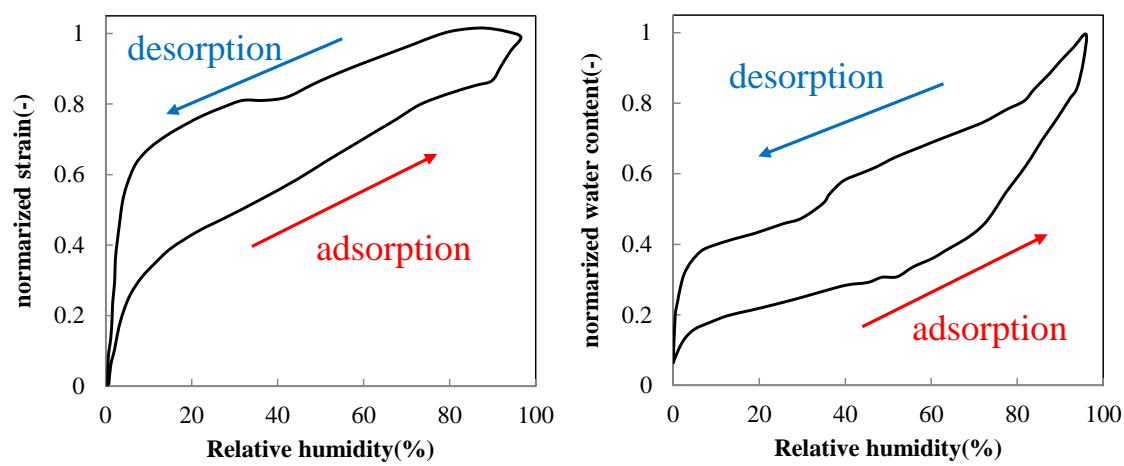

Figure 7. Experimental data for (a) volume change and (b) water vapor isotherm published by Feldman and Sereda[,1968]

\section{CONCLUSION}

In this paper, we proposed a new mesoscopic model of volume change in porous materials having slit shape pores with thermodynamic approach. The shape of strain isotherm predicted by our thermodynamic theory for a slit pore that is consisted of two immobile flat plates differs from the experimental hysteresis loop of volume change for hardened cement paste. In contract, the slit-like pore model with two mobile plates can reproduce the experimental hysteresis loop of adsorption/desorption isotherm and volume change for hardened cement paste, although the magnitude of strain become larger compared to experimental data.

\section{REFERENCES}

Andre Nonat(2004), The structure and stoichiometry of C-S-H, Cement and Concrete Research 34, 15211528

B. V. Deryagin and N. V. Churaev(1984), Wetting Films [in Russian], Nauka, Moscow

BV Deryagin, MM Kusakov(1936). "Properties of thin liquid layers" series Chemistry

C.G.V. Burgess, D.H. Everett, S(1989)."Nuttall, Adsorption hysteresis in porous materials"

Pure Appl. Chem. 61, 1845-1852.

F. Feldman and P. J. Sereda(1968), A model for hydrated portland cement paste as deduced from sorptionlength change and mechanical properties, Materiale de constructii : organ al Ministerului Industrializarii Lemnului si Materialelor de Constructii 1, 509.

P.Shiller, Th.Bier, M.Wahab and H.-J. Mogel (2008). "Mesoscopic model of volume changes due to moisture variations in porous materials." Colloids and Surfaces A: Physicochem. Eng.Aspects 327, 34 43

Reinhold Badmann, Norbert Stockhausen and Max Josef Setzer

S. Marcelja, N. Radic(1976), Repulsion of interfaces due to boundary water, Chem.

Phys. Lett. 42 129-130.

Reinhold Badmann(1981), Norbert Stockhausen, and Max Joseff Seter. "The Statistical Thickness and the Chemical Potential of Adsorbed Water Films" Journal of Colloid and Science 82, No. 2 534-542

interface 